\title{
Visceral leishmaniasis/human immunodeficiency virus co-infection in India: the focus of two epidemics
}

\author{
Purva Mathur, J. C. Samantaray, Madhu Vajpayee and Palash Samanta \\ Division of Parasitology and HIV/Immunology, Department of Microbiology, All India Institute of \\ Medical Sciences, Ansari Nagar, New Delhi - 110029, India
}

Correspondence

J. C. Samantaray

jsamantaray@yahoo.com

and jsamantaray@hotmail.com

Received 10 February 2006

Accepted 23 March 2006
India contributes heavily to the global burden of visceral leishmaniasis (VL, kala-azar) and human immunodeficiency virus (HIV)/AIDS. The prevalence of HIV seropositivity in VL patients at a tertiary care centre in northern India, as observed during a prospective study over a period of 2 years, is presented. Of the 104 cases of VL/post-kala-azar dermal leishmaniasis, six (5.7\%) were found to be HIV positive, compared to $11(5.5 \%)$ seropositive for HIV of 198 patients with fever due to other causes. Four of the six (67\%) VL/HIV co-infected patients had a chronic/relapsing course, not responding to antileishmanial treatment. A CD4 T-cell count of $<200 \mathrm{~mm}^{-3}$ was found in four of the five $(80 \%)$ co-infected patients in whom the test was done. Although the level of HIV/VL co-infection in the present study was lower than that of Mediterranean countries, there is a trend towards rising co-infection. The VL-endemic states of India have a huge population of migrant labourers, who work in high-HIV-prevalence states. The reported increase in the prevalence of HIV in the VL-endemic, populous states of India is a cause of grave concern, and co-infection may assume epidemic proportions in the coming decade if left unchecked.

\section{INTRODUCTION}

Visceral leishmaniasis (VL, kala-azar), a parasitic infection caused by protozoa of the genus Leishmania, is endemic in 66 countries. There is a growing number of reports of Leishmania/human immunodeficiency virus (HIV) coinfection across the world, the situation being particularly alarming in southern Europe, where $50-75 \%$ of adult cases of VL are HIV positive (Alvar et al., 1997; Lopez-Velez et al., 1998; Pintado \& Lopez-Velez, 2001; Pintado et al., 2001). Of the 45 million people infected by HIV worldwide, an estimated one-third live in the zones of endemic Leishmania infection (World Health Organization, 1995). India has the largest number of kala-azar cases (accounting for $40-50 \%$ of world disease burden) (Bora, 1999), and the second-largest HIV-infected population, accounting for approximately $10 \%$ of the global disease burden (USAID, 2003). The possible overlap in the distribution of VL and HIV in countries where both infections are highly endemic, such as India, may have grave consequences (Alvar et al., 1997).

Abbreviations: A/G ratio, albumin/globulin ratio; AllMS, All India Institute of Medical Sciences; BM, bone marrow; HIV, human immunodeficiency virus; LD body, Leishman-Donovan body; PKDL, post-kala-azar dermal leishmaniasis; VL, visceral leishmaniasis.

A map of the distribution of HIV and VL in India is available with the online version of this paper.
Although India contributes heavily to the global HIV and VL disease burdens, information about co-infection in this country is surprisingly sparse. Due to its implications in the diagnosis and management of VL, we conducted this study to ascertain the prevalence and clinico-epidemiological features of VL/HIV co-infection in a population endemic for $\mathrm{VL}$ in India.

\section{METHODS}

This prospective study was conducted at the department of Microbiology of All India Institute of Medical Sciences (AIIMS), New Delhi, from July 2003 to June 2005. Patients are referred from all over North India to AIIMS; its Microbiology department is the national HIV reference centre. The study was conducted after approval by the Institute Ethical Committee.

Patients. One hundred and four consecutive, parasitologically confirmed patients with kala-azar (96) and post-kala-azar dermal leishmaniasis (PKDL) (8) were included in the study. A detailed demographic and clinical history was recorded for each patient. The patients were also followed up during their hospital stay, with regular monitoring of their haemograms and biochemical profiles.

Controls. This group included 198 consecutive patients with fever, in whom kala-azar was a differential diagnosis. After detailed laboratory investigations [including a bone marrow (BM) examination], they were diagnosed to have aetiologies of fever other than VL.

HIV testing. The HIV antibody status was assessed by three ERS (ELISA, Rapid, simple) as recommended by the USA Centers for Disease Control and Prevention (Centers for Disease Control and 
Prevention, 2001). All tests were performed manually according to the manufacturer's recommendations. Pretest counselling was given to and informed consent taken from all patients.

\section{RESULTS}

During the 2 year period, a total of 104 cases of VL (96) and PKDL (8) were included in the study. Of the 96 cases of kala-azar, 94 (98\%) were from India [82 (85\%) from the VL endemic states; $12(12.5 \%)$ from non-endemic states] and two $(2 \%)$ were from Nepal. The age of the patients ranged from 6 months to 68 years (median 31 years); 76 (79\%) were males. Children accounted for $26(27 \%)$ of all VL patients. All except two patients had fever, the duration of which ranged from $<1$ month to 1.5 years (median 4.5 months); 92 (96\%) had splenomegaly, 81 (84\%) had pancytopenia and $93(97 \%)$ had reversal of albumin/ globulin $(A / G)$ ratio. All the eight cases of PKDL in the study were from the Bihar state of India. Six of the eight $(75 \%)$ patients were males, the age ranging from 15 to 28 years (median 21 years). All of them gave a history of having suffered from VL in the past.

Of the 104 cases of kala-azar/PKDL, six $(5 \cdot 7 \%)$ were found to be HIV positive. The details of these co-infected patients are presented in Table 1 .

Of the six cases of VL/HIV co-infection, patient 1 had been a known case of HIV infection for 4 years, and had successfully been treated for VL 1 year earlier. He was diagnosed to have a relapse of $\mathrm{VL}$ on the basis of a BM aspirate positive for Leishman-Donovan (LD) bodies. His splenomegaly regressed slightly after a course of amphotericin B; however, he expired after 7 months. Patient 2 had been diagnosed to have VL twice during the past 1 year, and was treated with repeated courses of sodium stibogluconate and miltefosine, to which he did not respond. Upon referral to our hospital, his BM was still positive for LD bodies, and he was also found to be seropositive for HIV. Even after a course of amphotericin B, his splenomegaly persisted. Patient 3 was diagnosed to have VL and HIV during the hospital stay, and was cured after a course of amphotericin B. Patient 4 presented with a history of recurrent fever for 4 years, and had been diagnosed and treated for VL twice during the past 4 years. He was diagnosed to be co-infected with HIV and $\mathrm{VL}$ at our hospital. Patient 5 was from a hilly, non-endemic region. Following a positive rK39 antibody test for VL, a BM aspirate was done which was positive for $\mathrm{LD}$ bodies. After a course of amphotericin B, his fever subsided, but splenomegaly persisted. The last patient presented with a history of chronic diarrhoea and weight loss, and had suffered from VL the previous year. Even after a course of amphotericin B, his fever persisted, and his BM was positive for LD bodies.

During the study, sera from 198 patients who presented clinically with symptoms like those of kala-azar were sent to us to rule out VL. BM aspirates of all these patients were negative for LD bodies. Amongst these, 150 (76\%) were from the VL-endemic areas of India and Nepal, and 48 $(24 \%)$ were from non-endemic areas of India. These patients had fever, 182 (92\%); splenomegaly, 131 (66\%);

Table 1. Profile of the Leishmania/HIV co-infected patients

Abbreviations: Sod. stibo, sodium stibogluconate; Ampho B, Amphotericin B; ICT, immunochromatographic test. -, negative; +, positive.

\begin{tabular}{|c|c|c|c|c|c|c|}
\hline \multirow{2}{*}{$\begin{array}{l}\text { Epidemiological/ } \\
\text { clinical/laboratory } \\
\text { findings }\end{array}$} & \multicolumn{6}{|c|}{ Patient } \\
\hline & 1 & 2 & 3 & 4 & 5 & 6 \\
\hline State of residence & Bihar & Bihar & Bihar & Bihar & Uttaranchal & Bihar \\
\hline Fever & - & + & + & + & + & + \\
\hline Hepatosplenomegaly & + & + & + & + & + & + \\
\hline Other symptoms & Diarrhoea & Diarrhoea & None & $\begin{array}{c}\text { Gross } \\
\text { weight loss }\end{array}$ & $\begin{array}{l}\text { Diarrhoea, } \\
\text { weight loss }\end{array}$ & $\begin{array}{l}\text { Gross weight } \\
\text { loss, diarrhoea }\end{array}$ \\
\hline $\begin{array}{l}\text { Pancytopenia/A/G reversal/ } \\
\text { positive aldehyde test }\end{array}$ & + & + & + & + & + & + \\
\hline rK39 ICT & + & + & - & + & + & + \\
\hline BM microscopy & + & + & + & + & + & + \\
\hline Mode of HIV acquisition & Heterosexual & Heterosexual & Heterosexual & Unknown & Heterosexual & Heterosexual \\
\hline $\mathrm{CD}^{+}$counts & 23 & 120 & 160 & Not done & 320 & 180 \\
\hline Relapse/non-response & + & + & - & + & - & + \\
\hline Other infections & Tuberculosis & None & None & Malaria & Tuberculosis & Tuberculosis \\
\hline Treatment given & $\begin{array}{c}\text { Ampho B } \\
\text { (twice) }\end{array}$ & $\begin{array}{l}\text { Sod. stibo, } \\
\text { Ampho B, } \\
\text { Miltefosin }\end{array}$ & Ampho B & $\begin{array}{l}\text { Ampho B } \\
\text { (twice) }\end{array}$ & Ampho B & Ampho B \\
\hline
\end{tabular}


weight loss, 79 (40\%); pancytopenia, 51 (26\%); and four ( $2 \%)$ had reversal of A/G ratio. Eleven $(5 \cdot 5 \%)$ of these 198 patients (nine from the VL endemic areas and two from non-endemic areas) were found to be HIV positive.

\section{DISCUSSION}

Leishmania/HIV co-infection has been reported from 33 countries to date (Sinha et al., 2003). Although India has a large population of both kala-azar cases and HIV-infected individuals, the prevalence of co-infection in our study was lower $(5 \cdot 7 \%)$ than that of Mediterranean countries. However, our findings are similar to those of a few other studies done in the VL-endemic state of Bihar. Before 2002, except for a single case, Leishmania/HIV co-infection was not documented in Bihar (Thakur et al., 2000, 2003). Subsequently, two studies from the same region have reported 1.5 and $6.3 \%$ of VL cases positive for HIV, respectively (Thakur et al., 2003; Sinha et al., 2003). Thus, there is a trend towards a gradually increasing prevalence of co-infection in our country. In southern Europe, on the other hand, $50-75 \%$ of adult patients of VL are HIV infected (Pintado \& Lopez-Velez, 2001; Pintado et al., 2001). The comparatively lower co-infection rates in our study can be explained by the differences in epidemiology of HIV and leishmaniasis in India and the Mediterranean countries.

In the Mediterranean countries, which were hypo-endemic for leishmaniasis, a steep rise in co-infection of VL and HIV was noted in the 1990s. This was primarily due to a geographical overlap of the two infections and the proposed shared mode of transmission by intravenous drug use in this area (Alvar et al., 1997). By contrast, in India, VL occurs only in four eastern states of the country, whereas the highprevalence states for HIV (antenatal HIV prevalence $>1 \%$ ) are the southern and north-eastern states (Bora, 1999; USAID, 2003). Thus, the geographical distribution of HIV and VL in India do not currently overlap (Supplementary Fig. S1). Further, in India, VL is primarily transmitted by sandfly bites, and HIV is primarily transmitted heterosexually, except for the north-eastern states (where intravenous drug users are the main risk group for HIV transmission and from which VL is not reported) (USAID, 2003). Reactivation of latent Leishmania infection has also been hypothesized as a reason for the upsurge of co-infection in southern Europe (Alvar et al., 1997). In India, Leishmania infects the majority of the population in the endemic regions during childhood, and most of the infections in adults are assumed to be due to reactivation. Therefore, an increase in HIV prevalence in these states, as seen in recent years, can precipitate an acute epidemic of VL/HIV co-infection if left unchecked.

A chronic, relapsing course was seen in $67 \%$ of co-infected patients in our study, which is the most outstanding feature of VL associated with HIV (Pintado \& Lopez-Velez, 2001). BM microscopy and immunochromatographic tests for rK-39 antibodies were found to be highly sensitive for diagnosis of VL in the HIV-infected patients of our study. However, a larger number of patients needs to be studied to validate the performance of rapid rK39 dipsticks in HIV-infected patients in our country.

The VL-hyperendemic state of Bihar, which is one of the populated and poor states of the country, provides $>70 \%$ of India's migrant labour population, and is classified as a highly HIV-vulnerable state by the National AIDS Control Organization, India (http://www.nacoonline.org/ facts.htm). However, the situation with respect to the HIV epidemic in the north-eastern and southern states of India is alarming, and a large number of labourers from Bihar have migrated to these states (Sinha et al., 2003; http://www. nacoonline.org/facts.htm). In India, migrant labourers are one of the main carriers of HIV, and Bihar has begun to receive back a large number of probable HIV patients because of its peripatetic labour force. To conclude, the Leishmania/HIV co-infection levels in India are currently lower than those of southern Europe. However, continuous surveillance is needed to ascertain any shift in the epidemiology of co-infection in this highly mobile and vulnerable population.

\section{ACKNOWLEDGEMENTS}

This work was partially funded by a grant from the Council of Scientific and Industrial Research (CSIR). We acknowledge the support provided by the technical staff of the Parasitology and HIV/Immunology laboratories of the department of Microbiology, AIIMS, New Delhi.

\section{REFERENCES}

Alvar, J., Canavate, C., Gutierrez-Solar, B. Jimenez M., Laguna, F., Lopez-Velez, R., Molina, R. \& Moreno, J. (1997). Leishmania and Human Immunodeficiency Virus coinfection: the first 10 years. Clin Microbiol Rev 10, 298-319.

Bora, D. (1999). Epidemiology of visceral leishmaniasis in India. Natl Med J India 12, 62-68.

Centres for Disease Control and Prevention (2001). Revised guidelines for HIV counseling, testing and referral. Morb Mortal Wkly Rep 50, RR19, 1-58.

Lopez-Velez, R., Jose, A., Molina, P., Guerrero, A., Baquero, F. \& Villarrubia, J. (1998). Clinicoepidemiologic characteristics, prognostic factors and survival analysis of patients coinfected with Human Immunodeficiency Virus and Leishmania in an area of Madrid, Spain. Am J Trop Med Hyg 58, 436-443.

Pintado, V. \& Lopez-Velez, R. (2001). HIV-associated visceral leishmaniasis. Clin Microbiol Infect 7, 291-300.

Pintado, V., Martin-Rabadan, P., Rivera, M., Moreno, S., Bouza, E. (2001). Visceral leishmaniasis in Human Immunodeficiency Virus (HIV)-infected and non HIV infected patients. Medicine 80, 54-73.

Sinha, P. K., Rabidas, V. N., Pandey, K., Verma, N., Gupta, A. K., Ranjan, A. \& Das, B. (2003). Visceral leishmaniasis and HIV coinfection in Bihar, India. J Acq Immun Def Syndr 32, 115-116.

Thakur, C. P., Sinha, P. K., Singh, R. K., Hassan, S. M. \& Narain, S. (2000). Miltefosine in a case of visceral leishmaniasis with HIV 
co-infection and rising incidence of this disease in India. Trans $R$ Soc Trop Med Hyg 94, 696-697.

Thakur, C. P., Narayan, S. \& Ranjan, A. (2003). Kala-azar (visceral leishmaniasis) and HIV coinfection in Bihar, India: is this combination increasing? J Acq Immun Def Syndr 32, 512-513.
USAID (2003). HIV/AIDS country profile, India, March. http:// www.usaid.gov/our_work/global_health/aids/countries/ane/India_05.pdf World Health Organization (1995). Report on the Consultative Meeting on Leishmania/HIV Co-infection. Report no. WHO/LEISH/ 95.35, pp. 1-14. Geneva: World Health Organization. 